\title{
Comparative transcriptome analysis of the gills and hepatopancreas from Macrobrachium rosenbergii exposed to the heavy metal Cadmium ( $\mathrm{Cd}^{2+}$ )
}

\section{Xue Liu}

Ministry of Agriculture (Shanghai Ocean University), Shanghai Ocean University)

Hucheng Jiang

Freshwater Fisheries Research Institute of Jiangsu Province

\section{Baoqing Ye}

Temasek Life Sciences Laboratory

\section{Hongli Qian}

Ministry of Agriculture (Shanghai Ocean University), Shanghai Ocean University)

\section{Ziqi Guo}

Ministry of Agriculture (Shanghai Ocean University), Shanghai Ocean University)

Haotian Bai

Ministry of Agriculture (Shanghai Ocean University), Shanghai Ocean University)

Jinhua Gong

Dinghe Aquatic Science and Technology Development Co., LTD

Jianbin Feng

Ministry of Agriculture (Shanghai Ocean University), Shanghai Ocean University)

Keyi Ma ( $D$ kyma@shou.edu.cn )

Ministry of Agriculture (Shanghai Ocean University), Shanghai Ocean University)

\section{Research Article}

Keywords: Metal tolerance, Cadmium (Cd2+), stress, RNA-Seq, Macrobrachium rosenbergii

Posted Date: April 8th, 2021

DOl: https://doi.org/10.21203/rs.3.rs-386333/v1

License: (c) (1) This work is licensed under a Creative Commons Attribution 4.0 International License.

Read Full License

Version of Record: A version of this preprint was published at Scientific Reports on August 9th, 2021. See the published version at https://doi.org/10.1038/s41598-021-95709-w. 


\section{Abstract}

Heavy metal Cadmium $\left(\mathrm{Cd}^{2+}\right)$ pollution has become a severe environmental problem for aquatic organisms. In crustaceans, gills and hepatopancreas play a vital role in the toxicology. However, in Macrobrachium rosenbergill, there are few researches about gill and hepatopancreases responding to $\mathrm{Cd}^{2+}$ stress at a molecular level. In this study, transcriptomic analysis was applied to characterize gene expression profiles of gills and hepatopancreas of $M$. rosenbergill after $\mathrm{Cd}^{2+}$ exposure for $0 \mathrm{~h}, 3 \mathrm{~h}$ and $3 \mathrm{~d}$. Six cDNA libraries (Gi 0 h, Gi 3 h, Gi 3 d, Hp 0 h, Hp 3 h, and Hp 3 d) were constructed and a total of 66,676 transcripts and 48,991 unigenes were annotated. Furthermore, differentially expressed genes (DEGs) were isolated by comparing the $\mathrm{Cd}^{2+}$ treated time-point libraries ( $3 \mathrm{~h}$ and $3 \mathrm{~d}$ group) with the control library ( $0 \mathrm{~h}$ group). The results showed that most of the DEGs were down-regulated after $\mathrm{Cd}^{2+}$ exposure and the number of DEGs among gill groups were significantly higher than those among hepatopancreas groups. GO functional and KEGG pathway analysis suggested many key DEGs in response to the $\mathrm{Cd}^{2+}$ stress, such as metallothionein and Hemocyanin. Additionally, a total of six DEGs were randomly selected to further identify their expressional profile by qPCR. The results indicated that these DEGs were involved in the response to $\mathrm{Cd}^{2+}$. This comparative transcriptome provides valuable molecular information on the mechanisms of responding to $\mathrm{Cd}^{2+}$ stress in $\mathrm{M}$. rosenbergii, which lays the foundation for further understanding of heavy metals stress.

\section{Introduction}

Over the past decades, aquatic heavy-metal pollution, such as copper $\left(\mathrm{Cu}^{2+}\right), \mathrm{Zinc}\left(\mathrm{Zn}^{2+}\right)$, and cadmium $\left(\mathrm{Cd}^{2+}\right)$, has become one of the greatest concerns for aquatic environmental bio-monitoring worldwide ${ }^{1}$ due to its high toxicity, non-degradability, and subsequent bioaccumulation and biomagnification ${ }^{2-5}$. $\mathrm{Cu}^{2+}$ is an essential metal that participates in normal physiological process in crustaceans. But, the high concentrations of $\mathrm{Cu}^{2+}$ could induce the generation of reactive oxygen species (ROS), which lead to oxidative damage in many organisms ${ }^{6,7} . \mathrm{Zn}^{2+}$ is highly toxic for aquatic crustaceans and ubiquitous heavy metal in aquatic environment. High concentrations of $\mathrm{Zn}^{2+}$ inhibiting oxygen consumption had been reported in Litopenaeus vannamei ${ }^{8}$, Litopenaeus schmitti ${ }^{9}$, and Farfantepenaeus paulensis ${ }^{10}$. Among heavy metal pollutants, $\mathrm{Cd}^{2+}$ pollution ranks first in the world ${ }^{11}$, and it is toxic even at a very low concentration ${ }^{12}$. $\mathrm{Cd}^{2+}$ causes the accumulation of ROS which induce oxidization of biological macromolecules and results in various physiological damages to animal tissues and organs ${ }^{13,14}$. Additionally, $\mathrm{Cd}^{2+}$, a non-essential and potentially toxic metal, can be accumulated in humans via food chain ${ }^{15}$. Previous researches displayed that lots of genes were differentially expressed after $\mathrm{Cd}^{2+}$ exposure in many crustaceans, such as Sinopotamon henanense ${ }^{16}$ and Eriocheir sinensis ${ }^{17}$. Hence, it is essential to focus on the potential response mechanism caused by $\mathrm{Cd}^{2+}$ stress in crustaceans.

As an important respiratory organ, the gill is likewise involved in ion transport, acid-base balance and osmoregulation in crustaceans ${ }^{18,19}$. Due to the crustacean gills exposing to the water in which they live, 
the gills play a vital role in the toxicology interactions, such as with heavy metals ${ }^{20}$. Furthermore, the hepatopancreas, a sensitive organ similar to the liver of higher organisms, is susceptible to be damaged by waterborne pollutants in crustaceans ${ }^{21-23}$. Therefore, gills and hepatopancreas are model organs for studying the response to heavy metal stress in crustaceans.

The giant freshwater prawn, Macrobrachium rosenbergii, is an important commercial prawn and widely cultured in China and other Pacific Rim countries ${ }^{24}$. As a freshwater cultured species, the prawn is susceptible to metals accumulation. Previous studies showed that structural changes of gills and hepatopancreas of $M$. rosenbergii could be caused by the $\mathrm{Cu}^{2+}$ accumulation, and the degree of damage observed was relevant to the elevated waterborne copper concentration ${ }^{21}$. Additionally, transcriptomic analysis of gills of $M$. rosenbergii showed that 19,417 and 8,989 differentially expressed genes (DEGs) were identified at $3 \mathrm{~h}$ and $48 \mathrm{~h}$ after $\mathrm{Cu}^{2+}$ exposure, respectively ${ }^{25}$, revealing that a large number of genes were involved in response to $\mathrm{Cd}^{2+}$ stress. Further research showed that the accumulation of $\mathrm{Cd}^{2+}$ also manifested histopathological changes in the gills and hepatopancreas of $M$. rosenbergii under $\mathrm{Cd}^{2+}$ exposure, and $\mathrm{Cd}^{2+}$ levels in tissues followed the order of: gills $>$ hepatopancreas ${ }^{26}$. To date, however, limited researches were focused on the $\mathrm{Cd}^{2+}$-related stress response and regulatory gene in $M$. rosenbergii.

In this study, transcriptome sequencing of gills and hepatopancreas in M. rosenbergii was performed to analyze transcriptional responses under $\mathrm{Cd}^{2+}$ pollution. Many vital genes in response to the $\mathrm{Cd}^{2+}$ were identified. The study provided valuable and reliable data for aquaculture and environmental monitoring management, and elucidated the potentially toxicological mechanism in $M$. rosenbergii

\section{Results}

\section{Transcriptome sequencing and functional gene annotation}

Six cDNA libraries were constructed for Illumina sequencing and the sequencing generated 47,932,697, 45,863,583, 46,324,011, 44,082,407, 43,621,438, and 46,621,968 clean reads for Gi 0 h, Gi 3 h, Gi 3 d, Hp 0 h, Hp 3 h, and Hp 3 d, respectively (Table 2). The clean reads were assembled, and generated 66,676 transcripts, which were further clustered into 48,991 unigenes. The unigenes comprised of $74,217,621$ bases, and the average length, largest length and smallest length were $1514.92 \mathrm{bp}, 36,523 \mathrm{bp}$ and $201 \mathrm{bp}$, respectively (Table 3 ). A length distribution of the total number of transcripts and unigenes is shown in Fig. 1. It is clearly displayed that there were 30,034 contigs (61.31\%) ranging from 201 to $1,000 \mathrm{bp}, 15,172$ contigs (30.97\%) ranging from 1,001 to $4,500 \mathrm{bp}$, and 3,785 contigs (7.73\%) over 4,500 bp in length. Likewise, there were 38,933 transcripts (33.16\%) ranging from 201 to 1,000 bp, 22,109 transcripts $(21.2 \%)$ ranging from 1,001 to $4,500 \mathrm{bp}$, and 5,634 transcripts ( $8.45 \%$ ) over $4,500 \mathrm{bp}$ in length.

We annotated all unigenes with reference to six functional databases (Fig. 2) and found that 14,812 (30.23\%), 11,869 (24.23\%), 16,093 (32.85\%), 18,536 (37.84\%), 14,384 (29.36\%) and 16,463 (33.6\%) unigenes were mapped to GO, KEGG, COG, NR, Swiss-Prot, and Pfam, respectively. 


\section{Identification Of Differentially Expressed Genes (DEGs)}

To identify genes displaying significant changes in expression level in the face of $\mathrm{Cd}^{2+}$ stress, we analyzed the expression level of each unigene by TPM method and found many DEGs by comparing the $\mathrm{Cd}^{2+}$ treated time-points libraries ( $3 \mathrm{~h}$ and $3 \mathrm{~d}$ group) with the control library ( $0 \mathrm{~h}$ group) (Supplementary Table S1). Compared to gill control group (Gi Oh), a total of 6,264 (2,010 upregulated and 4,254 downregulated) and 5,175 (2,186 upregulated and 2,989 downregulated) DEGs were identified in the Gi 3 h group and Gi 3 d group (Fig. 3), respectively. Long duration of $\mathrm{Cd}^{2+}$ exposure (Gi $3 \mathrm{~d}$ group) caused 4,222 genes to be differentially expressed compared with short duration (Gi 3 h group) (Fig. 3).

Furthermore, Venn analysis showed that 3,375 DEGs were changed at both time-points, while 2,889 DEGs were regulated just at Gi 3 h group and 1,800 genes were altered just at Gi 3 d group (Fig. 4). These timespecific genes might help to illustrate the stress response at different time-points. Similarly, many DEGs also existed between $\mathrm{Hp} \mathrm{O}$ h and $\mathrm{Hp} 3 \mathrm{~h}, \mathrm{Hp} \mathrm{O}$ h and $\mathrm{Hp} 3 \mathrm{~d}$, as well as $\mathrm{Hp} 3 \mathrm{~h}$ and $\mathrm{Hp} 3 \mathrm{~h}$ groups (Fig. 3 , 4). Additionally, the number of DEGs among gill groups were significantly higher than those among hepatopancreas groups.

\section{GO functional annotation and KEGG enrich analysis of DEGs}

To better understand the biological functions and gene interaction of DEGs, all DEGs (Gi $0 \mathrm{~h}$ vs Gi $3 \mathrm{~h}, \mathrm{Gi}$ $\mathrm{O}$ h vs $\mathrm{Gi} 3 \mathrm{~d}$, $\mathrm{Hp} \mathrm{O}$ h vs $\mathrm{Hp} 3 \mathrm{~h}$ and $\mathrm{Hp} \mathrm{O}$ vs $\mathrm{Hp} 3 \mathrm{~d}$ ) were annotated in $\mathrm{GO}$ terms (Fig. 5). Among the categories of biological process, cellular component, and molecular function, the top 2 enriched GO terms for each category were "cellular process and metabolic process", "membrane part and cell part", and "catalytic activity and binding”, respectively. Subsequently, KEGG pathway analysis was performed to identify the functions of DEGs and biological pathways involved in metal stress response. The top 20 significantly enriched KEGG pathways in each comparison are shown in Fig. 6. For the gill groups, 13 of those pathways (Intestinal immune network for IgA production, ECM-receptor interaction, Platelet activation, Cardiac muscle contraction, Retrograde endocannabinoid signaling, Focal adhesion, PI3K-Akt signaling pathway, Non-alcoholic fatty liver disease (NAFLD), Oxidative phosphorylation, Parkinson disease, Thermogenesis, Huntington disease, and Alzheimer disease) were enriched in both Gi $3 \mathrm{~h}$ and $\mathrm{Gi}$ $3 \mathrm{~d}$ compared with Gi $0 \mathrm{~h}$ group. whereas for the hepatopancreas groups, only 4 pathways (Glycosaminoglycan degradation, Starch and sucrose metabolism, Amino sugar and nucleotide sugar metabolism, and Glycerolipid metabolism) were enriched in both $\mathrm{Hp} 3 \mathrm{~h}$ and $\mathrm{Hp} 3 \mathrm{~d}$ compared with $\mathrm{Hp} 0$ h group. "Ribosome (272 genes)”,'Alzheimer disease (165 genes)”, “NOD-like receptor signaling pathway (21 genes)", and "lysosome (52 genes)" were significantly the most gene enriched pathways in Gi $0 \mathrm{~h}$ vs $\mathrm{Gi} 3$ h, Gi 0 h vs Gi 3 d, Hp 0 h vs Hp 3 h, and Hp 0 h vs Hp 3 d comparisons, respectively. These enriched pathways may play important roles in metal stress response. GO functional and KEGG analyses of DEGS (Gi 3 h vs Gi 3 d and Hp 3 h and Hp 3 d) are shown in Supplementary Figure S1.

\section{Validation of DEGs by qPCR}

The general trend of six DEGs (MT, Hemo, IFRD1, Hsp 67B2, Zbed4, and GH) involved in metal transportation and stress response was in accordance with the results from RNA sequencing (Fig. 7). 
Obviously, the change range of expression level of many DEGs in gill treated group is greater than those in hepatopancreas treated group compared to control group. For example, the expression level of GH gene significantly increased by eight and seven times in gill at $3 \mathrm{~h}$ and $3 \mathrm{~d}$ group, respectively. In addition, the DEGs present various expression patterns in gill and hepatopancreas after $\mathrm{Cd}^{2+}$ exposure. For instance, the expression level of Hemo significantly increased in gill at $3 \mathrm{~h}$ and then decreased at $3 \mathrm{~d}$, but consistently increased in hepatopancreas under $\mathrm{Cd}^{2+}$ stress. In contrast, the expression level of Hsp67B2 gene significantly decreased in gill at $3 \mathrm{~h}$ and then increased at $3 \mathrm{~d}$, but consistently increased in hepatopancreas under $\mathrm{Cd}^{2+}$ stress.

\section{Discussion}

In crustaceans, the gill epithelium is generally regarded as a major organ of respiration and osmoregulation, and the first site to be exposed to environmental pollutants ${ }^{27}$. During waterborne exposure to heavy metals, gills act as a protective barrier between the internal and external environment 28. Waterborne heavy metals were initially absorbed into epithelium cells of gill and transported into hemolymph, and finally infiltrated into internal organs ${ }^{28}$. Hepatopancreas is usually considered as a vital target organ for heavy metal toxicity and other environmental stresses in crustaceans and plays a major role in metal storage and in the detoxification process ${ }^{29}$. Additionally, crustaceans increases metabolic efficiency by promoting the digestive enzyme activities in hepatopancreas in response to heavy metal. ${ }^{30}$. Therefore, the gill and hepatopancreas are considered as a good indicator of water quality, and a suitable model for studies of heavy metals pollution.

We analyzed DEGs by comparing the $\mathrm{Cd}^{2+}$ treated time-points libraries with the control library. The results showed that the number of down-regulated DEGs is larger than up-regulated DEGs (Figure. 3), indicating that gene expressions were mainly inhibited by $\mathrm{Cd}^{2+}$, which leads to impairments in $M$. rosenbergii. The results were similar with Sinopotamon henanense and Danio rerio under $\mathrm{Cd}^{2+}$ stress ${ }^{16,31}$. The number of DEGs among Gi groups were significantly higher than those among Hp groups (Figure. 3), suggesting that the gill has a stronger stress response than hepatopancreas in short time. Additionally, the number of DEGs in Gi groups decreased with the increment of exposure time, while in $\mathrm{Hp}$ groups, the number of DEGs increased with the increment of exposure time (Figure. 3 ). The above results might be attributed to the reason that the gill acts as the entry site and transient store organ of the heavy metal for a short period of expose time, and $\mathrm{Cd} 2+$ is gradually transferred from the gills to hepatopancreas via the haemolymph with the prolongation of exposure time ${ }^{32}$.

The DEGs present various expression patterns, as identified by qPCR (Figure. 7). In this study, the expression level of metallothionein (MT) was significantly increased at 3h, then decreased at 3d, which may be related to the accumulation of $\mathrm{Cd}^{2+}$. Many studies have shown that MT is critical to heavy metal detoxification ${ }^{33,34}$ in addition to storage of essential elements that are necessary for metalloenzymes 35,36 . Some studies have proven that the accumulation of heavy metal has significant time effects. For instance, in Oncorhynchus mykiss, $\mathrm{Cu}^{2+}$ uptake increased during the 1-2 $\mathrm{h}$ under radiolabelled copper 
expose, and after $2 \mathrm{~h}, \mathrm{Cu}^{2+}$ level significantly decreased in gill ${ }^{37}$. A similar tendency was found in Acrossocheilus fasciatus $^{38}$, in which the expression level of zinc-finger BED domain-containing protein (Zbed) was significantly decreased after exposure to $\mathrm{Cd}^{2+}$, which is also consistent with what has been observed in Mytilus galloprovincialis exposed to $\mathrm{Cu}^{2+39}$. In contrast, hemocyanin-like protein, a crucial immune protein in arthropods ${ }^{40-42}$, was significantly increased expression after exposure to $\mathrm{Cd}^{2+}$. Heavy metals are handled through separate metabolic pathways dependent on hemocyanin ${ }^{43}$. In addition, the expression level of heat shock proteins (Hsps), common stress-inducible proteins, has been known to increase expression under various stressors, such as oxidative, and heavy metals, and viral infections ${ }^{44-}$ 46. For instance, Hsp70, Hsp40, and Hsp105, were significantly up-regulated in Eubalaena glacialis exposed to $\mathrm{Cd}^{2+}$. Interestingly, in M. rosenbergii, Hsp67B2 was consistently decreased in the hepatopancreas for three days under $\mathrm{Cd}^{2+}$ exposure, suggesting that Hsp67B2 may be suppressed by $\mathrm{Cd}^{2+}$ in this prawn. On the other hand, the expression level of IFRD1 was consistently increased in hepatopancreas under $\mathrm{Cd}^{2+}$ stress, which was consistent with the high upregulation of this gene in hepatopancreas of $M$. rosenbergii after virus infection ${ }^{47}$. IFRD1 protein has been proved to be involved in the regulation of inflammatory responses ${ }^{48}$, indicating that the increased expression of IFRD1 is intended to cure inflammation caused by $\mathrm{Cd}^{2+}$.

Nevertheless, further study is required to illustrate the regulatory mechanism of $M$. rosenbergii after exposure to $\mathrm{Cd}^{2+}$. The degree of histological damage of the gills and hepatopancreas under different concentrations and exposure days of $\mathrm{Cd}^{2+}$ is worth exploring in future research. Additionally, the effects of $\mathrm{Cd}^{2+}$ on the mitochondrion structure of gill and on superoxide dismutase (SOD) activity still need to be investigated.

\section{Conclusion}

In conclusion, we successfully constructed comparative gill and hepatopancreas transcriptome datasets in $\mathrm{Cd}^{2+}$ treated group and control group of $M$. rosenbergii. Thereafter, 48,991 unigenes were functionally annotated and a series of DEGs were isolated after $\mathrm{Cd}^{2+}$ exposure. Based on $\mathrm{GO}$ functional and KEGG pathway analyses, many DEGs that are potentially relevant to immune responses, antioxidant, and detoxification were identified.

\section{Material And Methods}

\section{Collection and maintenance of prawns}

A total of nine female and nine male $M$. rosenbergii $(23 \pm 2.5 \mathrm{~g})$ individuals used in this experiment were collected from Dinghe Aquatic Science and Technology Development Co. LTD (Jiangsu, China) and transported back to our laboratory. The prawns were maintained at $26 \pm 2{ }^{\circ} \mathrm{C}$ in a $50-\mathrm{L}$ aerated aquarium for three days before treatment. All animals were handled in accordance with guidelines established by 
the Animal Experiments Ethics Committee of Shanghai Ocean University for the care and use of laboratory animals.

\section{Cadmium Exposure Experiment}

After temporary rearing, $\mathrm{CdCl}_{2}$ solution was added to the culture water and mixed immediately, so as to expose all the prawns to the $\mathrm{Cd}^{2+}(80 \mathrm{ug} / \mathrm{L})$ based on the 96 -hour LC50 of Cd in M. rosenbergii ${ }^{49}$. Every day, the prawns were fed and the water was renewed $50 \%$ to maintain water quality. Subsequently, the experimental prawns were anesthetized on ice and dissected. The gills $(\mathrm{Gi})$ and hepatopancreas $(\mathrm{Hp})$ were randomly sampled from six individuals (three males and three females) for each of the 3 time points: $0 \mathrm{~h}, 3 \mathrm{~h}$ and $3 \mathrm{~d}$, after Cadmium exposure, and stored at $-80^{\circ} \mathrm{C}$ immediately for the following RNA extraction.

\section{Library Construction And Gene Function Annotation}

Total RNA was extracted from gills and hepatopancreas using Trizol reagent (Invitrogen, USA). The purity and amount of 36 RNA samples were assessed by NanoDrop2000C, and RNA integrity was verified by agarose electrophoresis. The RNA integrity was assessed by Agilent 2100 (RIN number > 6.5). For each group, equal amount of RNA from six individuals were pooled to make a sample for library construction. Three replicates were conducted for each library for statistics and comparison. Subsequently, the mRNA was purified and submitted to synthesize cDNA. Finally, the resulting six libraries (Gi 0 h, Gi 3 h, Gi 3 d, Hp $\mathrm{O}$ h, Hp $3 \mathrm{~h}$, and Hp $3 \mathrm{~d}$ ) were sequenced on Illumina Hiseq platform at Maiorbio company (Shanghai, China). Raw reads were trimmed by deleting adapter, ploy- $\mathrm{N}$ and low-quality reads. Then, the remaining clean reads were assembled into longer contigs using Trinity. The longest transcripts of each gene were defined as unigenes. Assembled unigenes were annotated by comparison to six databases, including NCBI non-redundant protein database (NR), Swiss-Prot, Pfam, Cluster of Orthologous Groups of proteins (COG), Gene Ontology (GO), and Kyoto Encyclopedia of Genes and Genomes (KEGG). The RNA-seq data have been deposited in the NCBI database under the accession number PRJNA707962.

\section{Gene Expression Analysis}

Gene expression values were calculated using RSEM and measured as transcripts per kilobase per million mapped reads (TPM) for six cDNA library. DESeq2 (with parameters of: $p$-value $<0.05$ and fold-change $\geq$ 2) was applied for analysis of differential expressed genes (DEGs). The DEGs between the library pairs (Gi 0 h vs Gi 3 h, Gi 0 h vs Gi 3 d, Gi 3 h vs Gi 3 d Hp 0 h vs Hp 3 h, Hp 0 h vs Hp 3 d, and Hp 3 h vs Hp 3 d) were identified. The GO and KEGG functional classification were performed to identify which DEGs were significantly enriched in GO terms and metabolic pathways.

\section{Validation of DEGs expression profiles using quantitative real-time RT-PCR (qPCR)}

To validate the Illumina sequencing results, six pooled RNA samples originally used for transcriptome sequencing were analyzed by qPCR. Six randomly selected genes: metallothionein (MT), hemocyanin-like protein (Hemo), interferon-related developmental regulator 1 (IFRD1), heat shock protein 67B2 (Hsp 
67B2), zinc finger BED domain-containing protein 4 (Zbed4), and gamma-glutamyl hydrolase (GH), were amplified by specific primers (Table 1). QPCR mixture $(20 \mu \mathrm{L})$ contained $10 \mu \mathrm{L}$ of PCR Master with SYRB green, $1 \mu \mathrm{L} \mathrm{Cd}{ }^{2+}$ cDNA template $(10 \mathrm{ng} / \mathrm{ul}), 0.25 \mu \mathrm{L}$ of each primer $(10 \mathrm{uM})$, and $8.5 \mu \mathrm{L} \mathrm{H}_{2} \mathrm{O}$. The primers of $\beta$-actin were used as the internal control. The relative quantification of six genes was calculated by the $2-\triangle \triangle \mathrm{CT}$ method ${ }^{50}$. Analysis of qPCR results was performed in GraphPad Prism 8. All data were presented as means \pm SD.

\section{Declarations}

Acknowledgements. This research was supported by the Natural Science Foundation of China (project number 31902348).

Author Contributions. Keyi Ma and Xue Liu conceived and designed the experiments; Xue Liu and Hongli Qian performed the experiments; Jianbin Feng®Ziqi Guo, and Haotian Bai analyzed the data; Jianbin Feng, Hucheng Jiang, Baoqing Ye and Jinhua Gong contributed reagents/materials/analysis tools; Xue Liu and Keyi Ma wrote the paper.

Conflict of interest. Author Jinhua Gong was the general manager of Dinghe Aquatic Science and Technology Development Co., LTD. All authors declare that the research was conducted in the absence of any commercial or financial relationships

ARRIVE guidelines statement. This study was carried out in compliance with the ARRIVE guidelines Approval statement. All experimental protocols were approved by the Key Laboratory of Freshwater Aquatic Genetic Resources, Ministry of Agriculture, Shanghai Ocean University in this paper.

\section{References}

1 Idrus, F. A., Basri, M. M., Rahim, K. A. A., Abd Rahim, N. S. \& Chong, M. D. Concentrations of Cadmium, Copper, and Zinc in Macrobrachium rosenbergii (giant freshwater prawn) from natural environment. Bull. Environ. Contam. Toxicol. 100, 350-355, doi:10.1007/s00128-018-2270-3 (2018).

2 Borrego, J., Morales, J. A., de la Torre, M. L. \& Grande, J. A. Geochemical characteristics of heavy metal pollution in surface sediments of the Tinto and Odiel river estuary (southwestern Spain). Environ. Geol. 41, 785-796, doi:10.1007/s00254-001-0445-3 (2002).

3 Rasmussen, A. D. \& Andersen, O. Effects of cadmium exposure on volume regulation in the lugworm, Arenicola marina. Aquat. Toxicol. 48, 151-164, doi:10.1016/s0166-445x(99)00045-4 (2000).

4 Asuquo, F. E., Ewa-Oboho, I., Asuquo, E. F. \& Udo, P. J. Fish species used as biomarker for heavy metal and hydrocarbon contamination for cross river, nigeria. Environmentalist 24, 29-37, doi:10.1023/B:ENVR.0000046344.04734.39 (2004). 
5 Papagiannis, I., Kagalou, I., Leonardos, J., Petridis, D. \& Kalfakakou, V. Copper and zinc in four freshwater fish species from Lake Pamvotis (Greece). Environ. Int. 30, 357-362, doi:10.1016/j.envint.2003.08.002 (2004).

6 Zeeshan, M., Murugadas, A., Ghaskadbi, S., Rajendran, R. B. \& Akbarsha, M. A. ROS dependent copper toxicity in Hydra-biochemical and molecular study. Comp. Biochem. Physiol., Part C: Toxicol. Pharmacol. 185, 1-12, doi:10.1016/j.cbpc.2016.02.008 (2016).

7 Schwarz, J. A., Mitchelmore, C. L., Jones, R., O'Dea, A. \& Seymour, S. Exposure to copper induces oxidative and stress responses and DNA damage in the coral Montastraea franksi. Comp. Biochem. Physiol., Part C: Toxicol. Pharmacol. 157, 272-279, doi:10.1016/j.cbpc.2012.12.003 (2013).

8 Wu, J. P. \& Chen, H. C. Effects of cadmium and zinc on oxygen consumption, ammonium excretion, and osmoregulation of white shrimp (Litopenaeus vannamei). Chemosphere 57, 1591-1598, doi:10.1016/j.chemosphere.2004.07.033 (2005).

9 Barbieri, E. Use of oxygen consumption and ammonium excretion to evaluate the sublethal toxicity of cadmium and zinc on litopenaeus schmitti (burkenroad, 1936, crustacea). Water Environ. Res. 79, 641646, doi:10.2175/106143006x136775 (2007).

10 Barbieri, E. Effects of zinc and cadmium on oxygen consumption and ammonium excretion in pink shrimp ( Farfantepenaeus paulensis, Pérez-Farfante, 1967, Crustacea). Ecotoxicology 18, 312-318, doi:10.1007/s10646-008-0285-y (2009).

$11 \mathrm{Su}, \mathrm{H}$. et al. Transcriptome analysis of the ovary of beet armyworm Spodoptera exigua under different exposures of cadmium stress. Chemosphere 251, 126372, doi:10.1016/j.chemosphere.2020.126372 (2020).

12 Jones MM \& C, C. Cadmium, a unique metal. Toxicol. J. 62, 1-25 (1990).

13 Thévenod, F. Cadmium and cellular signaling cascades: To be or not to be? Toxicol. Appl. Pharmacol. 238, 221-239, doi:10.1016/j.taap.2009.01.013 (2009).

14 Revathi, P., Vasanthi, L. A. \& Munuswamy, N. Effect of cadmium on the ovarian development in the freshwater prawn Macrobrachium rosenbergii (De Man). Ecotoxicol. Environ. Saf. 74, 623-629, doi:10.1016/j.ecoenv.2010.08.027 (2011).

15 Gaspic, Z. K., Zvonaric, T., Vrgoc, N., Odzak, N. \& Baric, A. Cadmium and lead in selected tissues of two commercially important fish species from the Adriatic Sea. Water Res. 36, 5023-5028, doi:10.1016/s0043-1354(02)00111-2 (2002).

16 Sun, M., Li, Y. T., Liu, Y., Lee, S. C. \& Wang, L. Transcriptome assembly and expression profiling of molecular responses to cadmium toxicity in hepatopancreas of the freshwater crab Sinopotamon henanense. Sci. Rep. 6, 19405, doi:10.1038/srep19405 (2016). 
17 Tang, D., Guo, H., Shi, X. \& Wang, Z. Comparative transcriptome analysis of the gills from the chinese mitten crab (Eriocheir japonica sinensis) exposed to the heavy metal cadmium. Turk. J. Fish. Aquat. Sci. 20, 467-479, doi:10.4194/1303-2712-v20_6_05 (2020).

18 P., W. et al. The organization and control of metabolism in the crustacean gill. Comp. Biochem. Physiol. 33, 529-548, doi:10.1016/0010-406X(70)90369-5 (1970).

19 Henry, R. P., Lucu, C., Onken, H. \& Weihrauch, D. Multiple functions of the crustacean gill: osmotic/ionic regulation, acid-base balance, ammonia excretion, and bioaccumulation of toxic metals. Front, Physiol. 3, 431, doi:10.3389/fphys.2012.00431 (2012).

20 Agoes et al. Impact of copper on the structure of gills and epipodites of the shrimp Penaeus japonicus (Decapoda). J. Crustacean Biol. 2, 209-223, doi:10.2307/1549227 (1999).

$21 \mathrm{Li}, \mathrm{N}$. , Zhao, Y. \& Yang, J. Impact of waterborne copper on the structure of gills and hepatopancreas and its impact on the content of metallothionein in juvenile giant freshwater prawn Macrobrachium rosenbergii (Crustacea: Decapoda) (vol 52, pg 73, 2009). Arch. Environ. Contam. Toxicol 56, 811-812, doi:10.1007/s00244-009-9306-y (2009).

22 Vogt, G. Monitoring of environmental pollutants such as pesticides in prawn aquaculture by histological diagnosis. Aquaculture 67, 157-164, doi:10.1016/0044-8486(87)90021-4 (1987).

23 Baticados, M. C. L. \& Tendencia, E. A. Effects of gusathion a on the survival and shell quality of juvenile penaeus monodon. Aquaculture 93, 9-19, doi:10.1016/0044-8486(91)90201-H (1991).

24 Sung, H. H., Kao, W. Y. \& Su, Y. J. Effects and toxicity of phthalate esters to hemocytes of giant freshwater prawn, Macrobrachium rosenbergii. Aquat. Toxicol. 64, 25-37, doi:10.1016/s0166445x(03)00011-0 (2003).

25 Guo, H., Deng, M., Liang, J., Lu, W. \& Shen, Y. Gill transcriptome alterations in Macrobrachium rosenbergii under copper exposure. Chemosphere 233, 796-808, doi:10.1016/j.chemosphere.2019.06.025 (2019).

26 Kaoud, H. A. \& Rezk, A. Effect of exposure to cadmium on the tropical freshwater prawn Macrobrachium rosenbergii. Afr. J. Aquat. Sci 36, 253-260, doi:10.2989/16085914.2011.636899 (2011).

27 Burnett, L. E. \& McMahon, B. R. Facilitation of $\mathrm{CO} 2$ excretion by carbonic anhydrase located on the surface of the basal membrane of crab gill epithelium. Respir. Physiol. 62, 341-348, doi:10.1016/00345687(85)90089-1 (1985).

28 Agoes, S., Mireille, C. D., Paul, T. J. \& Guy, C. Impact of Copper on the Structure of Gills and Epipodites of the Shrimp Penaeus japonicus (Decapoda). Journal of Crustacean Biology, 209-223. 
29 White, S. L. \& Rainbow, P. S. Accumulation of cadmium by Palaemon elegans (Crustacea: Decapoda). Mar. Ecol. Prog. Ser. 32, 17-25, doi:10.3354/meps032017 (1986).

30 Xuan, R. et al. Oxygen consumption and metabolic responses of freshwater crab Sinopotamon henanense to acute and sub-chronic cadmium exposure. Ecotoxicol. Environ. Saf. 89, 29-35, doi:10.1016/j.ecoenv.2012.10.022 (2013).

31 Gao, M., Lv, M., Liu, Y. \& Song, Z. Transcriptome analysis of the effects of Cd and nanomaterialloaded Cd on the liver in zebrafish. Ecotoxicol. Environ. Saf. 164, 530-539, doi:10.1016/j.ecoenv.2018.08.068 (2018).

32 Bjerregaard, P. Influence of physiological condition on cadmium transport from haemolymph to hepatopancreas in Carcinus maenas. Mar. Biol. 106, 199-209, doi:10.1007/BF01314801 (1990).

33 Mouneyrac, C. et al. Trace-metal detoxification and tolerance of the estuarine worm Hediste diversicolor chronically exposed in their environment. Mar. Biol. 143, 731-744, doi:10.1007/s00227-0031124-6 (2003).

34 Sterenborg, I. \& Roelofs, D. Field-selected cadmium tolerance in the springtail Orchesella cincta is correlated with increased metallothionein mRNA expression. Insect Biochem. Mol. Biol. 33, 741-747, doi:10.1016/s0965-1748(03)00070-5 (2003).

35 Brouwer, M., Winge, D. R. \& Gray, W. R. Structural and functional diversity of coppermetallothioneins from the American lobster Homarus americanus. J. Inorg. Biochem. 35, 289-303, doi:10.1016/0162-0134(89)84018-8 (1989).

36 Viarengo, A. \& Nott, J. A. Mechanisms of heavy metal cation homeostasis in marine invertebrates. Comp. Biochem. Physiol., Part C: Toxicol. Pharmacol. 104, 355-372, doi:10.1016/0742-8413(93)90001-2 (1993).

37 Sappal, R. et al. Copper and hypoxia modulate transcriptional and mitochondrial functionalbiochemical responses in warm acclimated rainbow trout (Oncorhynchus mykiss). Environ. Pollut. 211, 291-306, doi:10.1016/j.envpol.2015.11.050 (2016).

38 Liu, G.-D. et al. Molecular cloning, characterization and expression analysis of metallothionein in the liver of the teleost Acrossocheilus fasciatus exposed to cadmium chloride. Environ. Toxicol. Pharmacol. 53, 1-9, doi:10.1016/j.etap.2017.03.020 (2017).

39 Gomes, T., Chora, S., Pereira, C. G., Cardoso, C. \& Bebianno, M. J. Proteomic response of mussels Mytilus galloprovincialis exposed to CuO NPs and Cu2+: An exploratory biomarker discovery. Aquat. Toxicol. 155, 327-336, doi:10.1016/j.aquatox.2014.07.015 (2014).

40 Yan, F. et al. Identification and agglutination properties of hemocyanin from the mud crab (Scylla serrata). Fish Shellfish Immunol. 30, 354-360, doi:10.1016/j.fsi.2010.11.004 (2011). 
41 Decker, H. et al. Minireview: Recent progress in hemocyanin research. Integr. Comp. Biol. 47, 631644, doi:10.1093/icb/icm063 (2007).

42 Decker, H. \& Jaenicke, E. Recent findings on phenoloxidase activity and antimicrobial activity of hemocyanins. Dev. Comp. Immunol. 28, 673-687, doi:10.1016/j.dci.2003.11.007 (2004).

43 Pedrini-Martha, V. et al. Responsiveness of metallothionein and hemocyanin genes to cadmium and copper exposure in the garden snail Cornu aspersum. J. Exp. Zool., Part A, 1-11, doi:10.1002/jez.2425 (2020).

44 Singh, M. P., Reddy, M. M. K., Mathur, N., Saxena, D. K. \& Chowdhuri, D. K. Induction of hsp70, hsp60, hsp83 and hsp26 and oxidative stress markers in benzene, toluene and xylene exposed Drosophila melanogaster: role of ROS generation. Toxicol. Appl. Pharmacol. 235, 226-243, doi:10.1016/j.taap.2008.12.002 (2009).

45 Gupta, S. C., Sharma, A., Mishra, M., Mishra, R. K. \& Chowdhuri, D. K. Heat shock proteins in toxicology: How close and how far? Life Sci. 86, 377-384, doi:10.1016/j.lfs.2009.12.015 (2010).

46 Singh, M. P. et al. Effects of co-exposure of benzene, toluene and xylene to Drosophila melanogaster. Alteration in hsp70, hsp60, hsp83, hsp26, ROS generation and oxidative stress markers. Chemosphere 79, 577-587, doi:10.1016/j.chemosphere.2010.01.054 (2010).

47 Arockiaraj, J. et al. First report on interferon related developmental regulator-1 from Macrobrachium rosenbergii: Bioinformatic analysis and gene expression. Fish Shellfish Immunol. 32, 929-933, doi:10.1016/j.fsi.2012.02.011 (2012).

48 Micheli, L. et al. Pc4/tis7/ifrd1 stimulates skeletal muscle regeneration and is involved in myoblast differentiation as a regulator of myod and nf-kb. J. Biol. Chem. 286, 5691-5707, doi:10.1074/jbc.M110.162842 (2010).

49 Kaoud, H. A. \& Rezk, A. Effect of exposure to cadmium on the tropical freshwater prawn Macrobrachium rosenbergii. African Journal of Aquatic Science 36, 253-260, doi:10.2989/16085914.2011.636899 (2011).

50 Livak, K. J. \& Schmittgen, T. D. Analysis of Relative Gene Expression Data using Real-Time Quantitative PCR. Methods 25, 402-408 (2002).

\section{Tables}


Table 1

Real-time quantitative PCR primers used in this study

\begin{tabular}{|lll|}
\hline Gene & Forward primer sequence (5'-3') & Reverse primer sequence (5'-3') \\
\hline -actin & CGACGGTCAGGTCATCACCA & ACGTCGCACTTCATGATGGA \\
\hline MT & ACTCAGATTTTCTCAGCACCA & CTGAAAAACGGAACAACATGA \\
\hline hemo & TTATGGTGCCCTCCACAACTT & TGAAGAATGCAGGATCACGAGT \\
\hline IFRD1 & TGCTCTTTGTTGCTTTCGGTC & CTCTCAATGGCTTCTGTCTCCTC \\
\hline Hsp67B2 & CTACGGGTCGAGGGAACTTGA & CGATTCCGCCCTTAGATTTTG \\
\hline Zbed4 & CTATGGCACTTAGATGGGGGA & GAAACAACACAGAAGGGCTCA \\
\hline GH & TGATGGAAAAGCCTAAGCGAG & AGTCTATGTCAATTATGCCCG \\
\hline
\end{tabular}


Table 2

Summary of the sequencing data

\begin{tabular}{|cccccccc}
\hline Sample & Raw reads & Clean reads & \multicolumn{2}{l}{ Error rate(\%) } & Q20(\%) & Q30(\%) & GC content(\%) \\
\hline Gi0h_1 & $44,957,318$ & $44,445,834$ & 0.027 & 97.26 & 92.19 & 41.35 \\
\hline Gi0h_2 & $46,589,346$ & $46,042,048$ & 0.027 & 97.27 & 92.22 & 41.23 \\
\hline Gi0h_3 & $53,907,710$ & $53,310,208$ & 0.0267 & 97.39 & 92.48 & 41.29 \\
\hline Gi3d_1 & $41,266,892$ & $40,659,896$ & 0.027 & 97.24 & 92.22 & 41.9 \\
\hline Gi3d_2 & $49,137,222$ & $48,468,382$ & 0.0276 & 97.02 & 91.68 & 42.05 \\
\hline Gi3d_3 & $49,034,690$ & $48,462,470$ & 0.0269 & 97.33 & 92.37 & 42.07 \\
\hline Gi3h_1 & $47,657,670$ & $47,101,762$ & 0.0271 & 97.23 & 92.12 & 41.6 \\
\hline Gi3h_2 & $47,197,648$ & $46,611,040$ & 0.0273 & 97.14 & 91.95 & 41.66 \\
\hline Gi3h_3 & $45,785,340$ & $45,259,232$ & 0.0267 & 97.41 & 92.54 & 41.69 \\
\hline Hp0h_1 & $45,812,168$ & $45,193,388$ & 0.0268 & 97.35 & 92.43 & 43.52 \\
\hline Hp0h_2 & $45,040,828$ & $44,414,802$ & 0.0272 & 97.17 & 92.03 & 43.62 \\
\hline Hp0h_3 & $43,200,852$ & $42,639,030$ & 0.0268 & 97.35 & 92.46 & 43.65 \\
\hline Hp3d_1 & $45,569,698$ & $44,981,812$ & 0.0266 & 97.41 & 92.63 & 45.05 \\
\hline Hp3d_2 & $45,701,380$ & $45,056,284$ & 0.0266 & 97.39 & 92.58 & 45.2 \\
\hline Hp3d_3 & $41,305,288$ & $40,826,218$ & 0.0267 & 97.39 & 92.51 & 45.33 \\
\hline Hp3h_1 & $48,708,074$ & $48,189,044$ & 0.0262 & 97.59 & 93 & 44.55 \\
\hline Hp3h_2 & $46,612,070$ & $46,115,142$ & 0.0262 & 97.56 & 92.92 & 44.72 \\
\hline Hp3h_3 & $46,196,806$ & $45,561,718$ & 0.0266 & 97.38 & 92.64 & 44.51 \\
\hline
\end{tabular}


Table 3

The quality and length statistics of the transcripts and unigenes.

\begin{tabular}{|lll|}
\hline Type & Unigene & Transcript \\
\hline Total number & 48,991 & 66,676 \\
\hline Total base & $74,217,621$ & $106,673,706$ \\
\hline Largest length (bp) & 36,523 & 36,523 \\
\hline Smallest length (bp) & 201 & 201 \\
\hline Average length (bp) & 1514.92 & 1599.88 \\
\hline N50 length (bp) & 3,093 & 3,223 \\
\hline E90N50 length (bp) & 2,640 & 2,530 \\
\hline
\end{tabular}

\section{Figures}

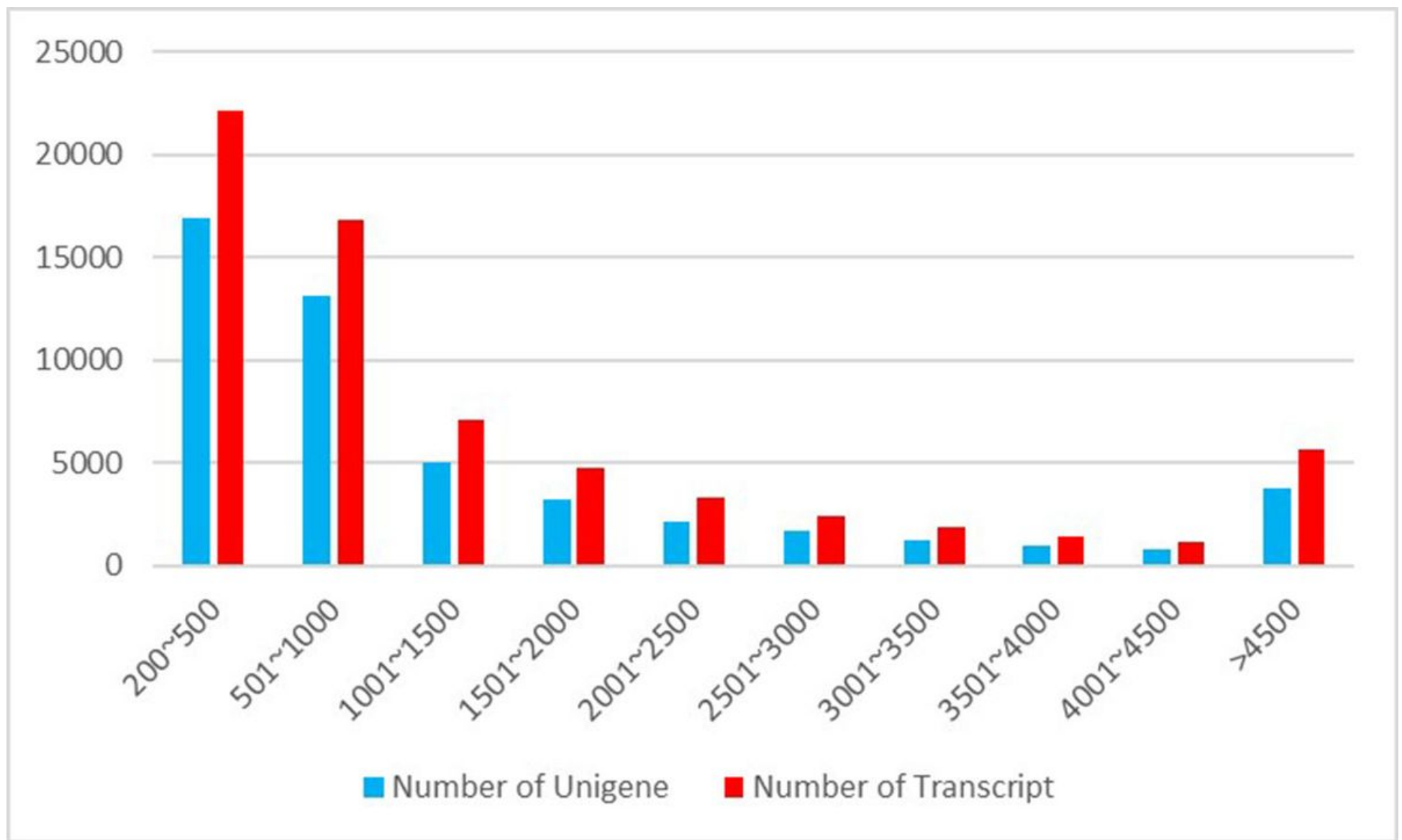

Figure 1 
Length distribution of transcripts and unigenes.

\section{Functional annotation of unigene}

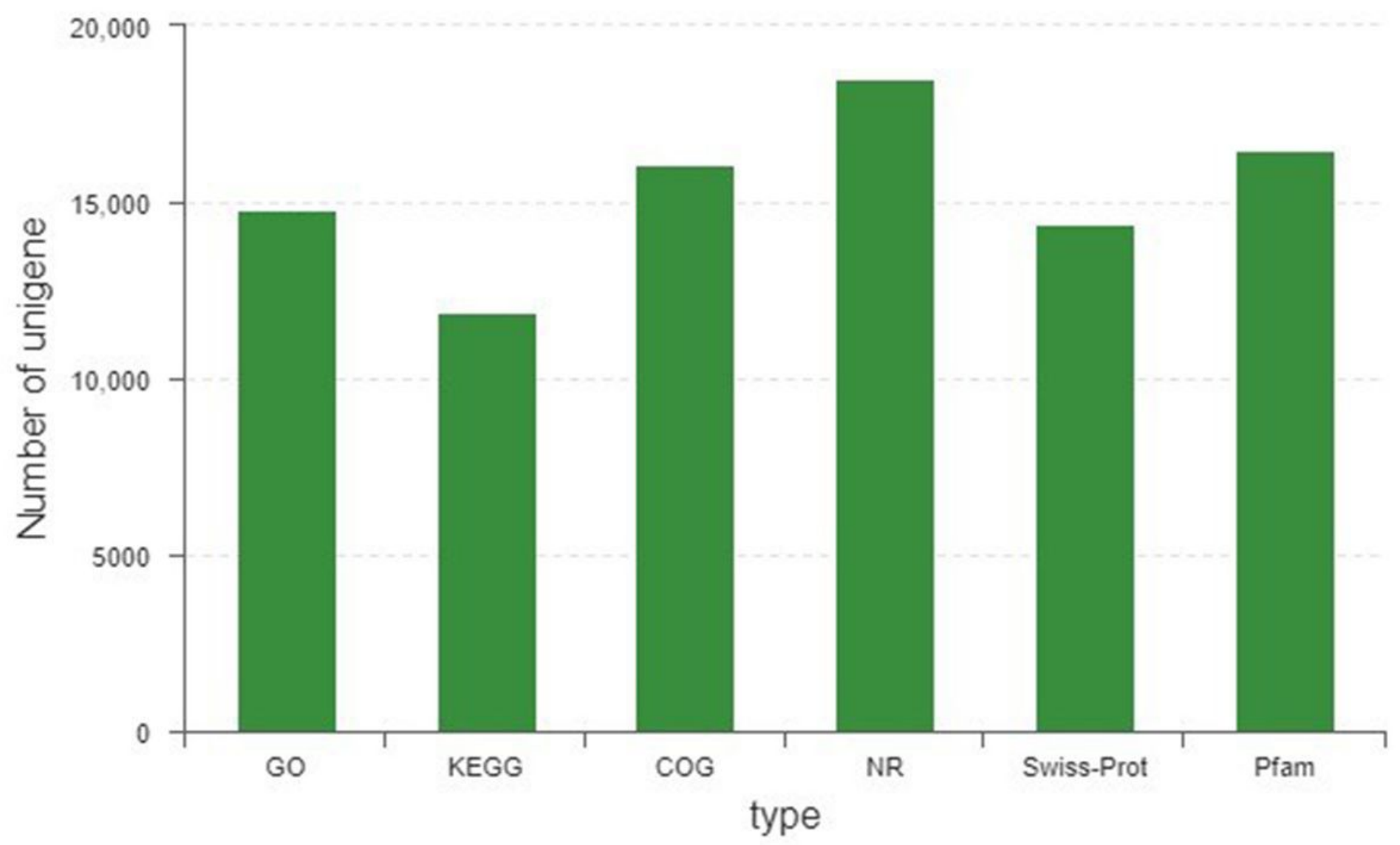

Figure 2

Annotation percentages of unigenes in different databases. 


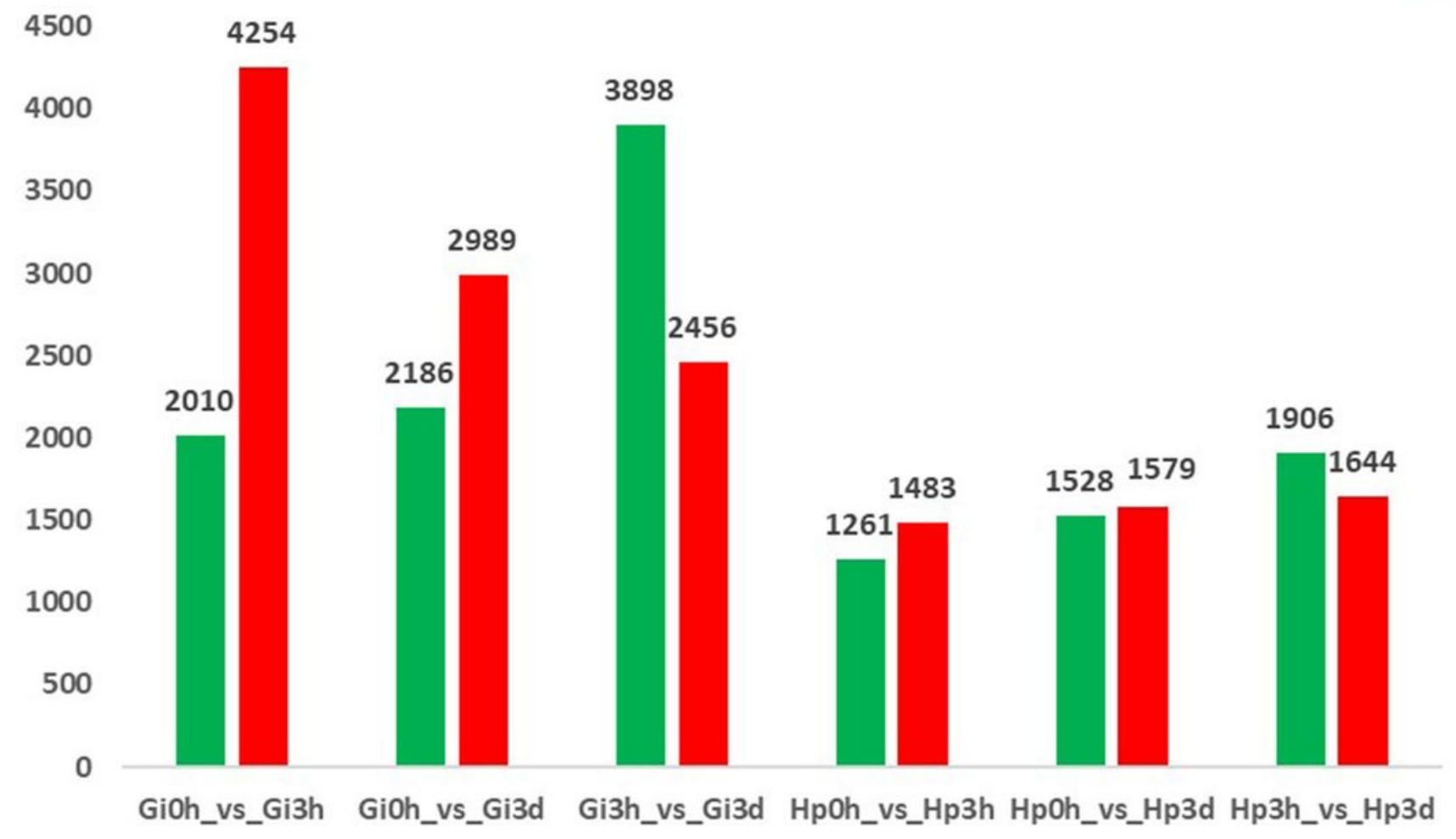

Figure 3

Differentially expressed genes (DEGs) between the sample pairs (Gi 0 h vs Gi 3 h, Gi 0 h vs Gi 3 d, Gi 3 h Vs Gi 3 d, Hp 0 h vs Hp 3 h , Hp 0 h vs Hp 3 d, and Hp 3 h vs Hp 3 d).

A
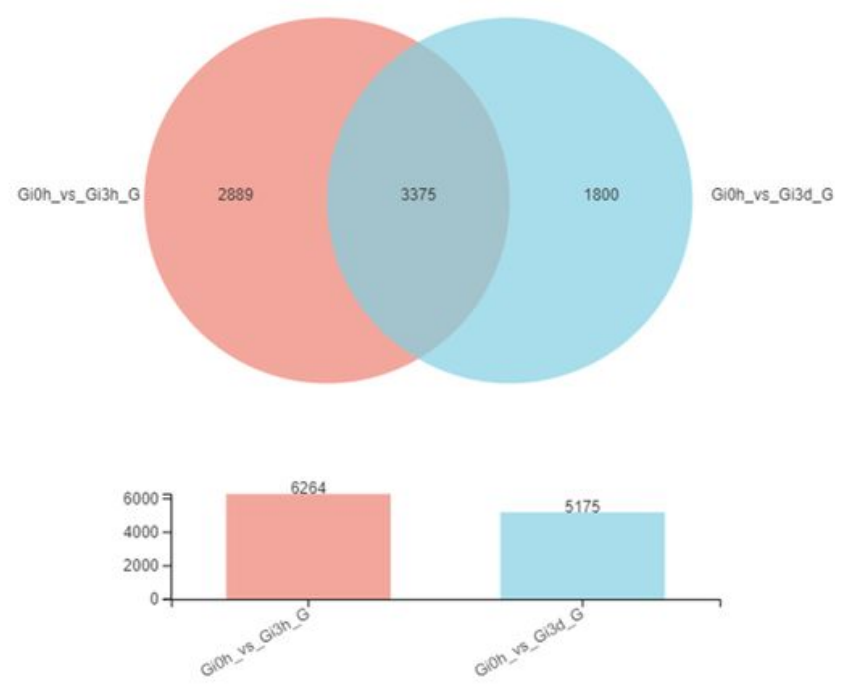

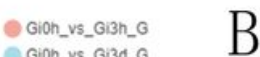

B
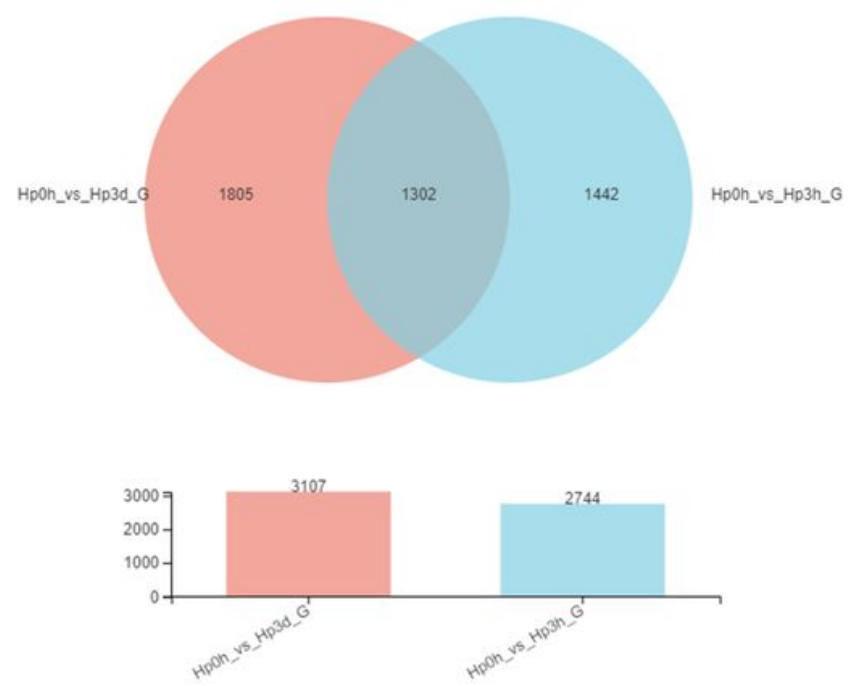

Figure 4 
Venn maps of DEGs between the sample pairs. (a) Venn diagrams showing the DEGs between $\mathrm{Gi} 3 \mathrm{~h}$ and $\mathrm{Gi} 3 \mathrm{~d}$. (b) Venn diagrams showing the DEGs between $\mathrm{Hp} 3 \mathrm{~h}$ and $\mathrm{Hp} 3 \mathrm{~d}$.

A

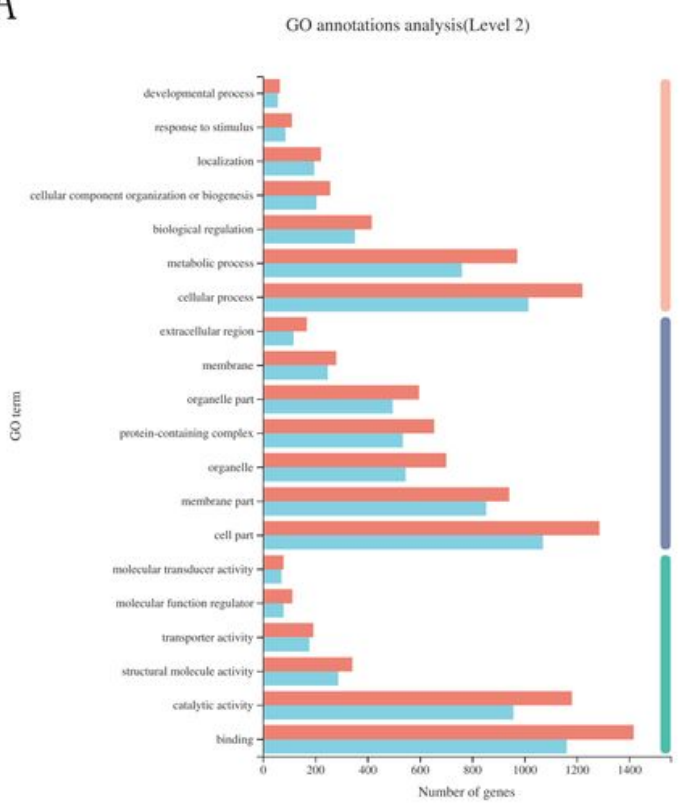

B

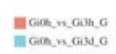

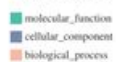

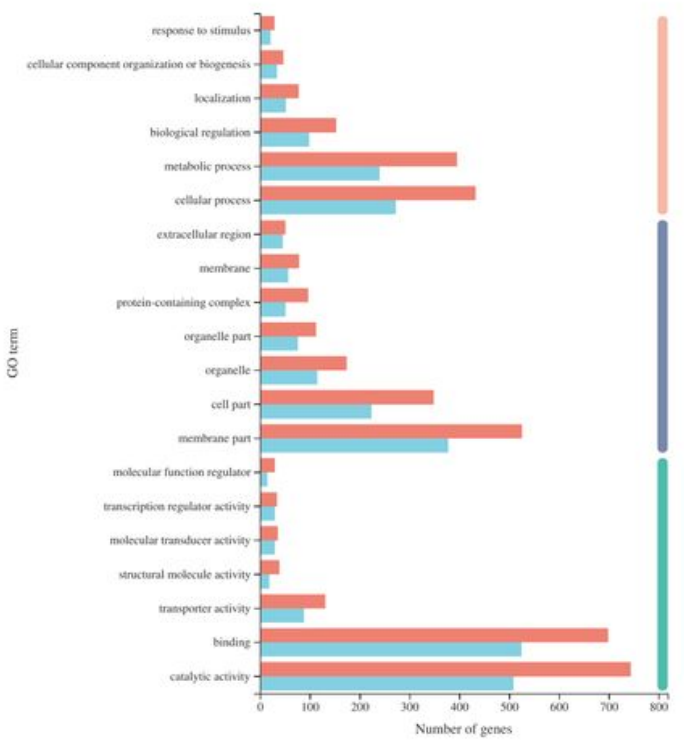

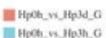

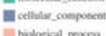

\section{Figure 5}

Gene ontology functional analyses of differentially expressed genes. (a) Gi 0 h vs Gi 3 h and Gi 0 h vs Gi 3 d. (b) $\mathrm{Hp} \mathrm{O}$ h vs Hp $3 \mathrm{~h}$ and $\mathrm{Hp} \mathrm{O}$ h vs Hp 3 d. 

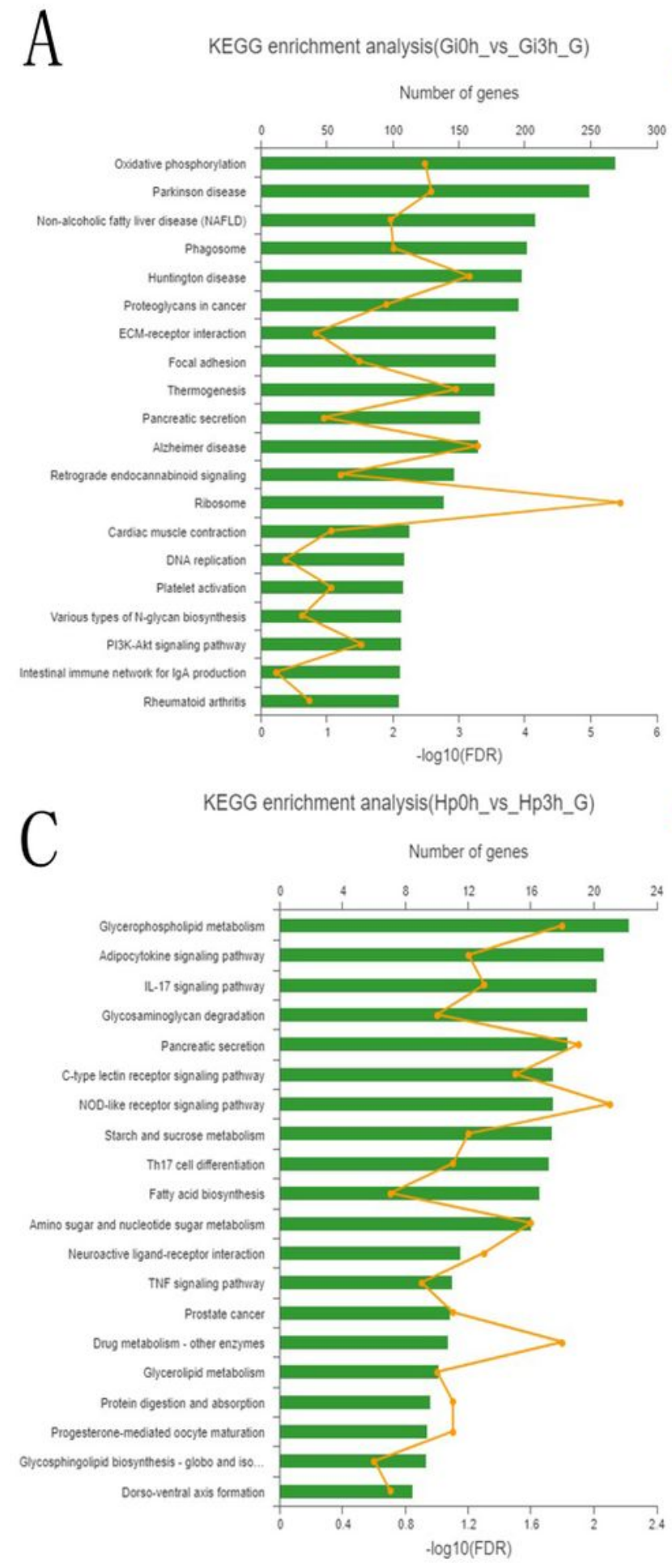

B

KEGG enrichment analysis(GiOh_vs_Gi3d_G)

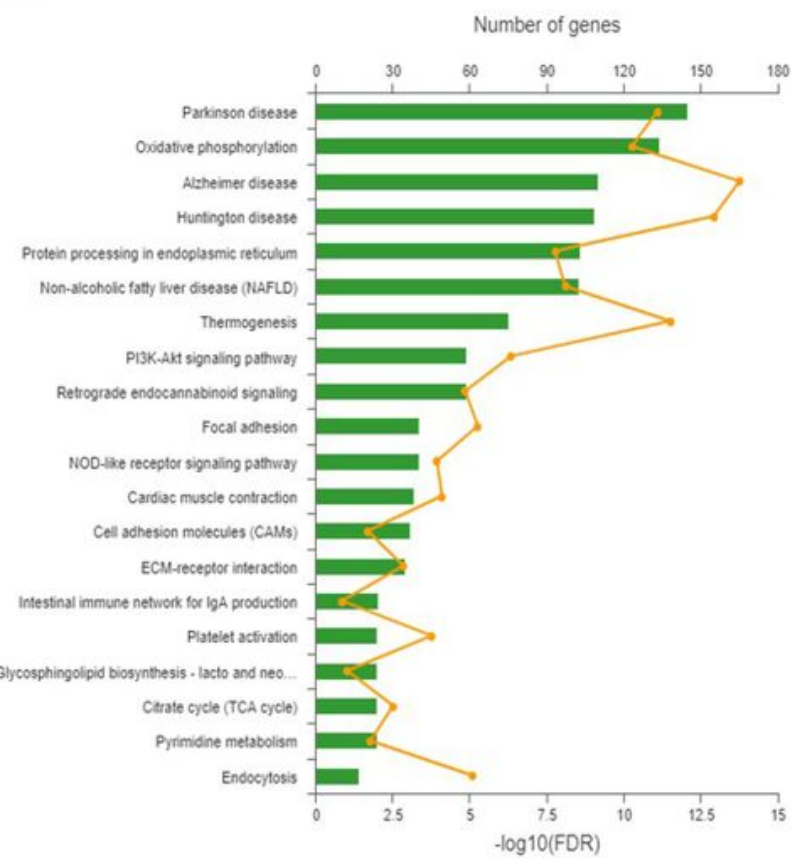

- $\log 10$ (FDR)
Number

KEGG enrichment analysis(HpOh_vs_Hp3d_G)

D

Number of genes

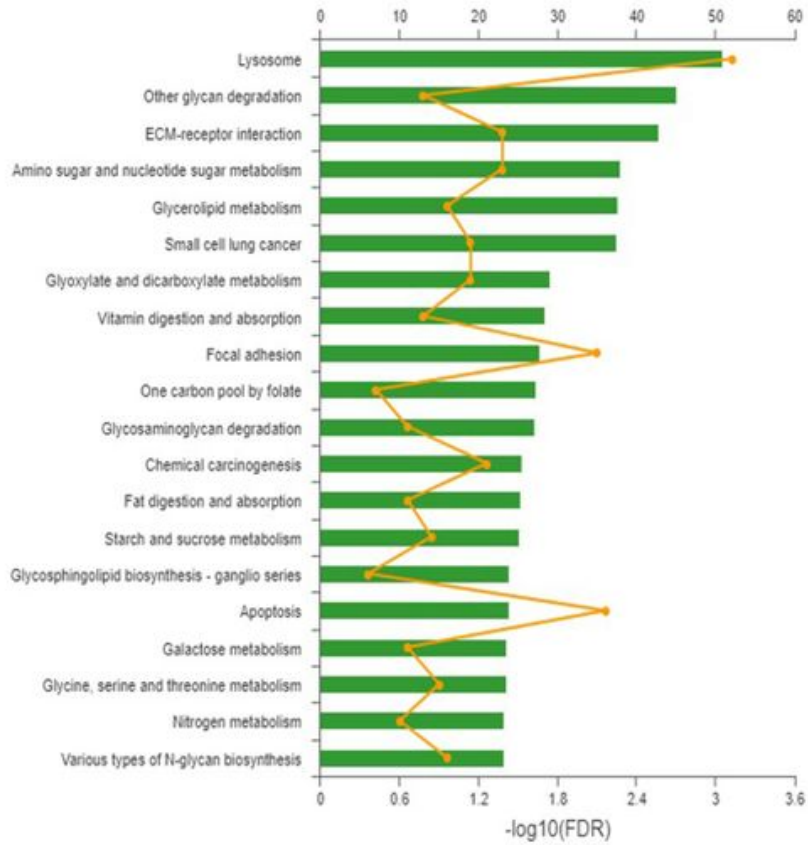

\section{Figure 6}

Kyoto Encyclopedia of Genes and Genomes functional analyses of differentially expressed genes between Gi $\mathrm{O}$ h and Gi $3 \mathrm{~h}$ (a), Gi $\mathrm{O}$ h and Gi 3 d (b), $\mathrm{Hp} \mathrm{O}$ h and $\mathrm{Hp} 3 \mathrm{~h}$ (c), $\mathrm{Hp} \mathrm{O}$ h and $\mathrm{Hp} 3 \mathrm{~d}$ (d). 
$\mathrm{GH}$

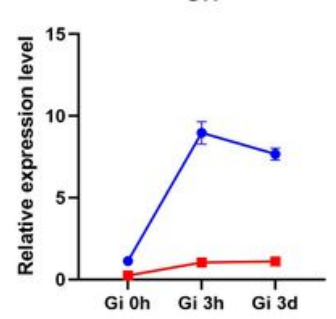

Hsp67B2
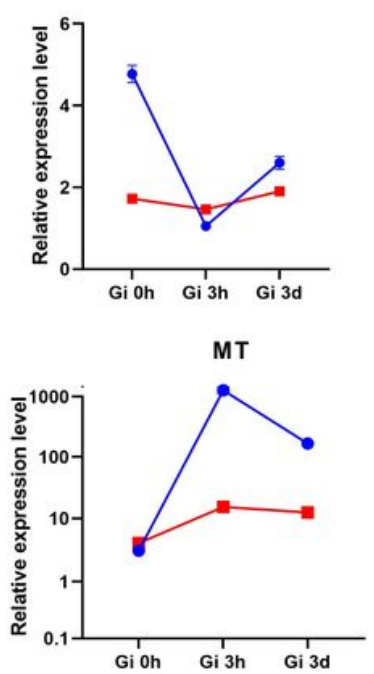

GH

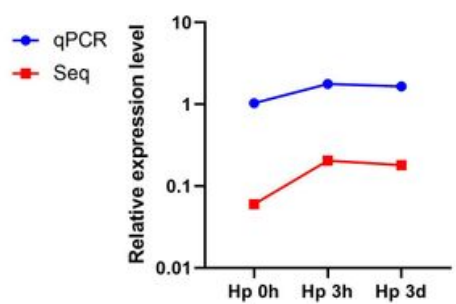

Hsp67B2
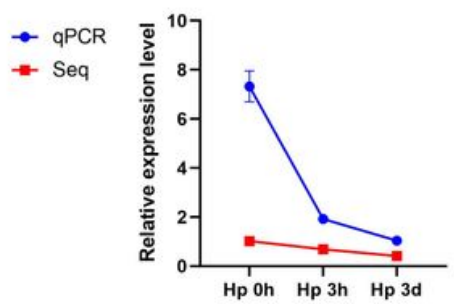

MT

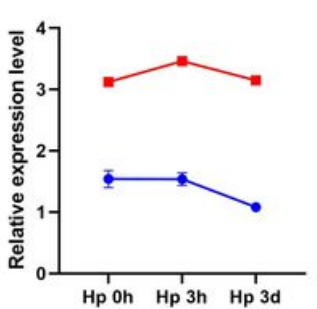

Hemo

- qPCR
- Seq

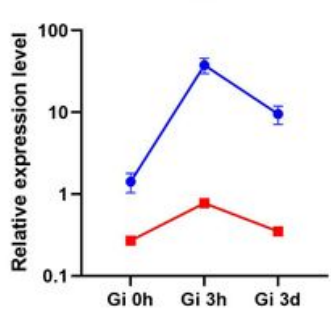

IFRD1

- qPCR
- Seq

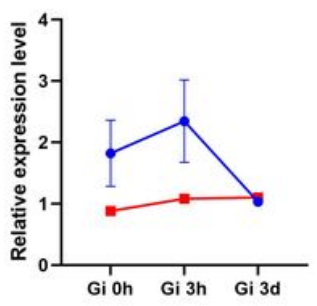

Zbed4

- qPCR
- Seq

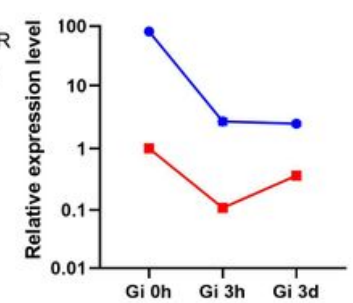

Hemo

$\rightarrow$ qPCR

- Seq

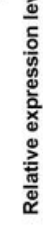

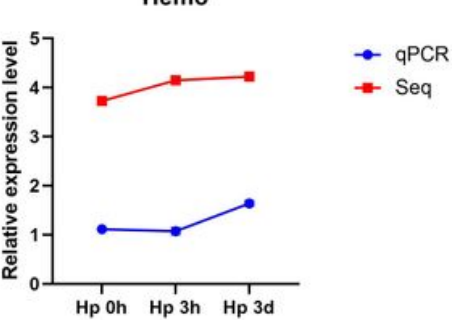

IFRD1
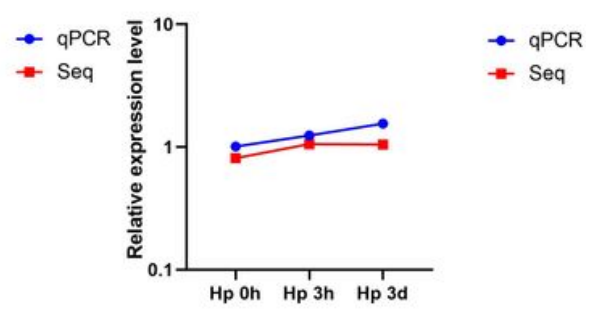

Zbed4

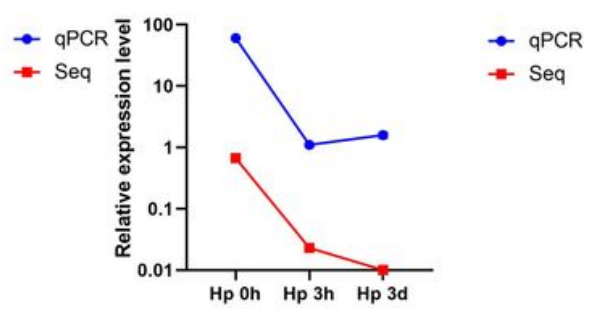

\section{Figure 7}

Validation of the relative expression level of six genes associated with $\mathrm{Cd} 2+$ stress using quantitative real time PCR. Blue bar represents the qPCR results, and red bar represents the RNA-Seq results. MT, metallothionein; Hemo, hemocyanin-like protein; IFRD1, interferon-related developmental regulator 1; Hsp67B2, heat shock protein 67B2-like; Zbed4, zinc finger BED domain-containing protein 4-like; and GH, gamma-glutamyl hydrolase-like.

\section{Supplementary Files}

This is a list of supplementary files associated with this preprint. Click to download.

- SupplementFigureS1.jpg

- SupplementTableS1.xls 